\title{
On the Exact Series Solution for Nonhomogeneous Strongly Coupled Mixed Parabolic Boundary Value Problems
}

\author{
Vicente Soler, ${ }^{1}$ Emilio Defez, ${ }^{2}$ Roberto Capilla, ${ }^{3}$ and José Antonio Verdoy ${ }^{2}$ \\ ${ }^{1}$ Departamento de Matemática Aplicada, Universitat Politècnica de València, Camino de Vera S/N, 46022 Valencia, Spain \\ ${ }^{2}$ Instituto de Matemática Multidisciplinar, Universitat Politècnica de València, Camino de Vera S/N, 46022 Valencia, Spain \\ ${ }^{3}$ Departamento de Ingeniería Electrónica, Universitat Politècnica de València, Camino de Vera S/N, 46022 Valencia, Spain
}

Correspondence should be addressed to Emilio Defez; edefez@imm.upv.es

Received 4 April 2014; Accepted 9 July 2014; Published 14 October 2014

Academic Editor: Natig M. Atakishiyev

Copyright (C) 2014 Vicente Soler et al. This is an open access article distributed under the Creative Commons Attribution License, which permits unrestricted use, distribution, and reproduction in any medium, provided the original work is properly cited.

An exact series solution for nonhomogeneous parabolic coupled systems of the type $u_{t}-A u_{x x}=G(x, t), A_{1} u(0, t)+B_{1} u_{x}(0, t)=$ $0, A_{2} u(l, t)+B_{2} u_{x}(l, t)=0,0<x<1, t>0, u(x, 0)=f(x)$, where $A_{1}, A_{2}, B_{1}$, and $B_{2}$ are arbitrary matrices for which the block matrix $\left(\begin{array}{cc}A_{1} & B_{1} \\ A_{2} & B_{2}\end{array}\right)$ is nonsingular, and $A$ is a positive stable matrix, is constructed.

\section{Introduction}

Coupled partial differential systems with coupled boundaryvalue conditions are frequent in different areas of science and technology, as in scattering problems in quantum mechanics [1-3], in chemical physics [4-6], coupled diffusion problems [7-9], thermo-elastoplastic modelling [10], and so forth. The solution of these problems has motivated the study of vector and matrix Sturm-Liouville problems; see [11-14], for example.

Recently, see $[15,16]$, an exact series solution for the homogeneous initial-value problem

$$
\begin{gathered}
u_{t}(x, t)-A u_{x x}(x, t)=0, \quad 0<x<1, t>0 \\
A_{1} u(0, t)+B_{1} u_{x}(0, t)=0, \quad t>0 \\
A_{2} u(1, t)+B_{2} u_{x}(1, t)=0, \quad t>0 \\
u(x, 0)=f(x), \quad 0 \leq x \leq 1,
\end{gathered}
$$

where $u=\left(u_{1}, u_{2}, \ldots, u_{m}\right)^{T}$ and $f(x)=$ $\left(f_{1}(x), f_{2}(x), \ldots, f_{m}(x)\right)^{T} \quad$ are $m$-dimensional vectors, was constructed under the following hypotheses and notations.
(1) The matrix coefficient $A$ is a matrix which satisfies the following condition:

$$
\operatorname{Re}(z)>0, \quad \forall z \in \sigma(A),
$$

where $\sigma(C)$ denotes the set of all the eigenvalues of a matrix $C$ in $\mathbb{C}^{m \times m}$. Thus, $A$ is a positive stable matrix (where $\operatorname{Re}(z)$ denotes the real part of $z \in \mathbb{C}$ ).

(2) Matrices $A_{i}, B_{i}, i=1,2$, are $m \times m$ complex matrices, and we assume that the block matrix

$$
\left(\begin{array}{ll}
A_{1} & B_{1} \\
A_{2} & B_{2}
\end{array}\right) \text { is regular }
$$

and also that the matrix pencil

$$
A_{1}+\rho_{0} B_{1} \text { is regular }
$$

that is, condition (4) involves the existence of some $\rho_{0} \in \mathbb{C}$, matrix $A_{1}+\rho_{0} B_{1}$ being invertible; see [17].

Using condition (4), we can introduce the following matrices $\widetilde{A}_{1}$ and $\widetilde{B}_{1}$ defined by

$$
\widetilde{A}_{1}=\left(A_{1}+\rho_{0} B_{1}\right)^{-1} A_{1}, \quad \widetilde{B}_{1}=\left(A_{1}+\rho_{0} B_{1}\right)^{-1} B_{1},
$$


which satisfy the condition $\widetilde{A}_{1}+\rho_{0} \widetilde{B}_{1}=I$, where matrix $I$ denotes, as usual, the identity matrix. Under hypothesis (3), it is easy to show that matrix $B_{2}-\left(A_{2}+\rho_{0} B_{2}\right) \widetilde{B}_{1}$ is regular (see [18] for details) and we can introduce matrices $\widetilde{A}_{2}$ and $\widetilde{B}_{2}$ defined by

$$
\begin{aligned}
& \widetilde{A}_{2}=\left[B_{2}-\left(A_{2}+\rho_{0} B_{2}\right) \widetilde{B}_{1}\right]^{-1} A_{2}, \\
& \widetilde{B}_{2}=\left[B_{2}-\left(A_{2}+\rho_{0} B_{2}\right) \widetilde{B}_{1}\right]^{-1} B_{2},
\end{aligned}
$$

that satisfy the conditions $\widetilde{B}_{2}-\left(\widetilde{A}_{2}+\rho_{0} \widetilde{B}_{2}\right) \widetilde{B}_{1}=I, \widetilde{B}_{2} \widetilde{A}_{1}-$ $\widetilde{A}_{2} \widetilde{B}_{1}=I$. Under the above assumptions, in [15], we have consider the following essential hypothesis:

exist $b_{1} \in \sigma\left(\widetilde{B}_{1}\right)-\{0\}, \quad b_{2} \in \sigma\left(\widetilde{B}_{2}\right), \quad v \in \mathbb{C}^{m}-\{0\}$, such that $\left(\widetilde{B}_{1}-b_{1} I\right) v=\left(\widetilde{B}_{2}-b_{2} I\right) v=0$,

if the vector valued function $f(x)$ satisfies hypotheses

$$
\begin{gathered}
f \in \mathscr{C}^{2}([0,1]) \\
\left(1-\rho_{0} b_{1}\right) f(0)+b_{1} f^{\prime}(0)=0 \\
-\left(\frac{1-b_{2}+\rho_{0} b_{1} b_{2}}{b_{1}}\right) f(1)+b_{2} f^{\prime}(1)=0,
\end{gathered}
$$

under the additional hypothesis

$$
\begin{aligned}
& f(x) \in \operatorname{Ker}\left(\widetilde{B}_{1}-b_{1} I\right) \cap \operatorname{Ker}\left(\widetilde{B}_{2}-b_{2} I\right), 0 \leq x \leq 1, \\
& \operatorname{Ker}\left(\widetilde{B}_{1}-b_{1} I\right) \cap \operatorname{Ker}\left(\widetilde{B}_{2}-b_{2} I\right)
\end{aligned}
$$

is an invariant subspace with respect to matrix $A$,

where a subspace $E$ of $\mathbb{C}^{m}$ is invariant by the matrix $A \in$ $\mathbb{C}^{m \times m}$, if $A(E) \subset E$, in order to construct an exact series solution $u(x, t)$ of homogeneous problem (1).

Moreover, in [16], under the above assumptions and replacing the condition (7) by the following hypothesis

$0 \in \sigma\left(\widetilde{B}_{1}\right), \quad a_{2} \in \sigma\left(\widetilde{A}_{2}\right)$, and we have $w \in \mathbb{C}^{m}-\{0\}$, so that $\widetilde{B}_{1} w=\left(\widetilde{A}_{2}-a_{2} I\right) w=0$,

if the vector valued function $f(x)$ satisfies the new hypotheses

$$
\begin{gathered}
f \in \mathscr{C}^{2}([0,1]) \\
f(0)=0 \\
a_{2} f(1)+f^{\prime}(1)=0,
\end{gathered}
$$

under the additional hypothesis

$$
\begin{aligned}
& f(x) \in \operatorname{Ker}\left(\widetilde{B}_{1}\right) \cap \operatorname{Ker}\left(\widetilde{A}_{2}-a_{2} I\right), \quad 0 \leq x \leq 1, \\
& \operatorname{Ker}\left(\widetilde{B}_{1}\right) \cap \operatorname{Ker}\left(\widetilde{A}_{2}-a_{2} I\right)
\end{aligned}
$$

is an invariant subspace respect to matrix $A$, then an exact series solution $u(x, t)$ of homogeneous problem (1) is constructed, see [16].

This paper deals with the construction of the exact series solution of the nonhomogeneous problem

$$
\begin{gathered}
u_{t}(x, t)-A u_{x x}(x, t)=G(x, t), \quad 0<x<1, t>0 \\
A_{1} u(0, t)+B_{1} u_{x}(0, t)=0, \quad t>0 \\
A_{2} u(1, t)+B_{2} u_{x}(1, t)=0, \quad t>0 \\
u(x, 0)=f(x), \quad 0 \leq x \leq 1 .
\end{gathered}
$$

We provide conditions for the vector valued function $G(x, t)$ in order to ensure the existence and convergence of a series solution of the problem (13)-(16).

Throughout this paper, we will assume the results and nomenclature given in $[15,16]$. If $B$ is a matrix in $\mathbb{C}^{m \times m}$, its 2-norm denoted by $\|B\|$ is defined by [19, page 56]

$$
\|B\|=\sup _{z \neq 0} \frac{\|B z\|_{2}}{\|z\|_{2}},
$$

where, for a vector $y$ in $\mathbb{C}^{m},\|z\|_{2}$ is the usual euclidean norm of $y$. Let us introduce the notation $\alpha(C)=\max \{\operatorname{Re}(z) ; z \in$ $\sigma(C)\}$. By [19, page 556], it follows that

$$
\left\|e^{t B}\right\| \leq e^{\alpha(B) t} \sum_{k=0}^{m} \frac{\|\sqrt{m} B\|^{k} t^{k}}{k !} .
$$

If $P_{m}(x)$ is a polynomial of degree $m$, then by fórmula 2.323 of [20, page 92$]$, one gets

$$
\int P_{m}(x) e^{a x} d x=\frac{e^{a x}}{a} \sum_{k=0}^{m}(-1)^{k} \frac{P_{m}^{(k)}(x)}{a^{k}} .
$$

We need to recall two well-known inequalities [21]:

(i) The Schwarz inequality: Let $a, b \in \mathbb{R}$ so that $a \leq b$; if $f$ and $g$ are continuous functions on $[a, b]$, then

$$
\int_{a}^{b} f(x) g(x) d x \leq\left(\int_{a}^{b} f(x)^{2} d x\right)^{1 / 2}\left(\int_{a}^{b} g(x)^{2} d x\right)^{1 / 2} .
$$

(ii) The Hölder inequality: If we consider the convergent series of positive terms $\sum_{n \geq 0} a_{n}$ and $\sum_{n \geq 0} b_{n}$, then

$$
\sum_{n \geq 0} a_{n}^{1 / 2} b_{n}^{1 / 2} \leq\left(\sum_{n \geq 0} a_{n}\right)^{1 / 2}\left(\sum_{n \geq 0} b_{n}\right)^{1 / 2} .
$$

The paper is organized as follows. In Section 2, the solution of (13)-(16) is obtained under hypothesis (7)-(9), and the convergence of the series solution for the problem, under these hypotheses (7)-(9), is studied. In Section 3, the solution of (13)-(16) is obtained under hypotheses (10)-(12) and the convergence of the series solution for the problem, under these hypotheses (10)-(12), is also studied. In Section 4, we will introduce an algorithm and give two illustrative examples. Conclusions are given in Section 5. 


\section{A Series Solution for Nonhomogeneous Problem (13)-(16) under Hypotheses (7)-(9). Convergence}

We suppose that the hypotheses (7)-(9) hold. We will find the solution of nonhomogeneous problem with homogeneous boundary conditions (13)-(16) where we will suppose that the vector valued function $G(x, t)$ satisfies the conditions that we will indicate to ensure the convergence of the solution proposal.

We will suppose that the vector valued function $G(x, t)$ satisfies conditions (8) replacing $f(x)$ by $G(x, t)$, and, therefore, $G(x, t)$ admits a series expansion of Sturm-Liouville eigenfunctions which are given by

$$
G(x, t)=X_{0}(x) T_{0}(t)+\sum_{\lambda_{n} \in \mathscr{F}} X_{n}(x) T_{n}(t),
$$

where the set of eigenvalues $\mathscr{F}$ are given by equation (27) of [15], with the positive roots $\lambda_{k} \in(k \pi,(k+1) \pi), k \geq 1$, of equation (16) of [15], to which is added the eigenvalue $\lambda_{0} \in$ $(0, \pi)$ if $\left(1-b_{2}+\rho_{0} b_{1} b_{1}\right)\left(1-\rho_{0} b_{1}\right) / b_{1}<1$, and, by equation (35) of [15], the eigenvalue 0 is also added if $1 \in \sigma\left(-\widetilde{A}_{2} \widetilde{A}_{1}\right)$, and the eigenfunctions are given by

$$
\begin{gathered}
X_{0}(x)=\alpha\left(\left(1-\rho_{0} b_{1}\right) x-b_{1}\right), \quad \alpha= \begin{cases}1, & 0 \in \mathscr{F} \\
0, & 0 \notin \mathscr{F}\end{cases} \\
X_{n}(x)=\left(1-\rho_{0} b_{1}\right) \operatorname{sen}\left(\lambda_{n} x\right)-b_{1} \lambda_{n} \cos \left(\lambda_{n} x\right)
\end{gathered}
$$

and coefficients

$$
\begin{aligned}
& T_{n}(t)=\frac{\int_{0}^{1} G(x, t) X_{n}(x) d x}{\int_{0}^{1} X_{n}^{2}(x) d x}, \\
& T_{0}(t)=\frac{\int_{0}^{1} G(x, t) X_{0}(x) d x}{\int_{0}^{1} X_{0}^{2}(x) d x},
\end{aligned}
$$

fulfilling the Bessel inequality; see [11, page 223] and [22]:

$$
\begin{aligned}
\sum_{\lambda_{n} \in \mathscr{F}}\left\|T_{n}(t)\right\|^{2}\left\|X_{n}(x)\right\|^{2} & =\sum_{\lambda_{n} \in \mathscr{F}}\left\|T_{n}(t)\right\|^{2} \int_{0}^{1} X_{n}^{2}(x) d x \\
& \leq \int_{0}^{1}\|G(x, t)\|^{2} d x .
\end{aligned}
$$

We know that the positive roots $\lambda_{k}, k \geq 1$ fulfill Lemma 1 of [15]; then,

$$
\lim _{k \rightarrow \infty} \lambda_{k}=\infty
$$

and taking into account that $\lambda_{k} \in(k \pi,(k+1) \pi), k \geq 1$, then the numerical series $\sum_{k \geq 1} 1 / \lambda_{k}^{2}$ is convergent.

Using the eigenfunction method, we will construct a formal solution of the problem (13)-(15) in the form

$$
u(x, t)=X_{0}(x) R_{0}(t)+\sum_{\lambda_{n} \in \mathscr{F}} e^{-\lambda_{n}^{2} A t} X_{n}(x) R_{n}(t),
$$

where

$$
\begin{array}{cc}
B_{n}(t)=\int_{0}^{t} e^{A \lambda_{n}^{2} s} T_{n}(s) d s, & B_{0}(t)=\int_{0}^{t} T_{0}(s) d s, \\
R_{n}(t)=B_{n}(t)+D_{n}, & R_{0}(t)=B_{0}(t)+D_{0} .
\end{array}
$$

Taking into account that $u(x, t)$ have to satisfy the initial condition (16), one gets that

$$
u(x, 0)=f(x)=X_{0}(x) R_{0}(0)+\sum_{\lambda_{n} \in \mathscr{F}} X_{n}(x) R_{n}(0) ;
$$

thus, as $f(x)$ satisfies (8), then it also admits a series expansion of Sturm-Liouville eigenfunctions:

$$
\begin{aligned}
& R_{n}(0)=D_{n}=\frac{\int_{0}^{1} f(x) X_{n}(x) d x}{\int_{0}^{1} X_{n}^{2}(x) d x}, \\
& R_{0}(0)=D_{0}=\frac{\int_{0}^{1} f(x) X_{0}(x) d x}{\int_{0}^{1} X_{0}^{2}(x) d x} .
\end{aligned}
$$

Note that we can write

$$
\begin{aligned}
u(x, t)= & X_{0}(x) R_{0}(t) \\
& +\sum_{\lambda_{n} \in \mathscr{F}} e^{-\lambda_{n}^{2} A t} X_{n}(x) R_{n}(t) \\
= & X_{0}(x) D_{0}+\sum_{\lambda_{n} \in \mathscr{F}} e^{-\lambda_{n}^{2} A t} X_{n}(x) D_{n} \\
& +X_{0}(x) B_{0}(t)+\sum_{\lambda_{n} \in \mathscr{F}} e^{-\lambda_{n}^{2} A t} X_{n}(x) B_{n}(t) \\
= & v(x, t)+w(x, t),
\end{aligned}
$$

where

$$
v(x, t)=X_{0}(x) D_{0}+\sum_{\lambda_{n} \in \mathscr{F}} e^{-\lambda_{n}^{2} A t} X_{n}(x) D_{n}
$$

is a solution of the homogeneous problem with homogeneous boundary conditions:

$$
\begin{gathered}
v_{t}(x, t)-A v_{x x}(x, t)=0, \quad 0<x<1, \quad t>0 \\
A_{1} v(0, t)+B_{1} v_{x}(0, t)=0, \quad t>0 \\
A_{2} v(1, t)+B_{2} v_{x}(1, t)=0, \quad t>0 \\
v(x, 0)=f(x), \quad t>0,
\end{gathered}
$$

the convergence of $v(x, t)$ has been studied previously in [15], and

$$
w(x, t)=X_{0}(x) B_{0}(t)+\sum_{\lambda_{n} \in \mathscr{F}} e^{-\lambda_{n}^{2} A t} X_{n}(x) B_{n}(t)
$$


is a solution of the nonhomogeneous problem with homogeneous boundary conditions:

$$
\begin{gathered}
w_{t}(x, t)-A w_{x x}(x, t)=G(x, t), \quad 0<x<1, t>0 \\
A_{1} w(0, t)+B_{1} w_{x}(0, t)=0, \quad t>0 \\
A_{2} w(1, t)+B_{2} w_{x}(1, t)=0, \quad t>0 \\
w(x, 0)=0, \quad t>0 .
\end{gathered}
$$

Now, we will study the convergence of the formal solution obtained in (27). Previously, we need to find a bound to the integral

$$
\int_{0}^{t}\left\|e^{-A \lambda_{n}^{2}(t-s)}\right\|^{2} d s
$$

Using (18), one gets that

$$
\begin{aligned}
& \left\|e^{-A \lambda_{n}^{2}(t-s)}\right\|^{2} \\
& \leq e^{-2 \alpha(A) \lambda_{n}^{2}(t-s)}\left(\sum_{k=0}^{m-1} \frac{\|\sqrt{m} A\|^{k}\left(\lambda_{n}^{2}(t-s)\right)^{k}}{k !}\right)^{2} \\
& =e^{-2 \alpha(A) \lambda_{n}^{2}(t-s)} P_{2 m-2}\left(\lambda_{n}^{2}(t-s)\right),
\end{aligned}
$$

where $P_{2 m-2}(x)$ is a polynomial of degree $2 m-2$ with positive coefficients. Thus,

$$
\int_{0}^{t}\left\|e^{-A \lambda_{n}^{2}(t-s)}\right\|^{2} d s \leq \int_{0}^{t} e^{-2 \alpha(A) \lambda_{n}^{2}(t-s)} P_{2 m-2}\left(\lambda_{n}^{2}(t-s)\right) d s .
$$

Performing the change of variable $v=\lambda_{n}^{2}(t-s)$ and thaking into account (19), we can write expression (39) in the form

$$
\begin{aligned}
& \int_{0}^{t} e^{-2 \alpha(A) \lambda_{n}^{2}(t-s)} P_{2 m-2}\left(\lambda_{n}^{2}(t-s)\right) d s \\
& =\frac{e^{-2 \alpha(A)}}{2 \lambda_{n}^{2} \alpha(A)}\left(L-\sum_{k=0}^{2 m-2} \frac{P_{2 m-2}^{(k)}\left(\lambda_{n}^{2} t\right)}{(2 \alpha(A))^{k}}\right),
\end{aligned}
$$

where

$$
L=\sum_{k=0}^{2 m-2} \frac{P_{2 m-2}^{(k)}(0)}{(2 \alpha(A))^{k}}
$$

and taking into account that the coefficients of $P_{2 m-2}^{(k)}(x)$ and $P_{2 m-2}^{(k)}(0)$ are positive, one gets from (39) and (40) that

$$
\int_{0}^{t}\left\|e^{-A \lambda_{n}^{2}(t-s)}\right\|^{2} d s \leq \frac{L}{2 \lambda_{n}^{2} \alpha(A)}, \quad t \geq 0 .
$$

Now, one gets that

$$
u(x, t)=v(x, t)+w(x, t)
$$

where $v(x, t)$ is a solution of problem (34), whose convergence has been studied in [15]; we will study the convergence of $w(x, t)$, solution of problem (36), defined by (35), where $B_{n}(t)$ are defined by (28). Taking norm and using (20), one gets that

$$
\begin{aligned}
\left\|\sum_{\lambda_{n} \in \mathscr{F}} e^{-\lambda_{n}^{2} A t} X_{n}(x) B_{n}(t)\right\|^{2} \\
=\left\|\sum_{\lambda_{n} \in \mathscr{F}} \int_{0}^{t} e^{-A \lambda_{n}^{2}(t-s)} T_{n}(s) X_{n}(x) d s\right\|^{2} \\
\leq\left(\sum_{\lambda_{n} \in \mathscr{F}}\left\|\int_{0}^{t} e^{-A \lambda_{n}^{2}(t-s)} T_{n}(s) X_{n}(x) d s\right\|\right)^{2} \\
\leq\left(\sum_{\lambda_{n} \in \mathscr{F}} \int_{0}^{t}\left\|e^{-A \lambda_{n}^{2}(t-s)} T_{n}(s) X_{n}(x) d s\right\|\right)^{2} \\
\leq\left(\sum_{\lambda_{n} \in \mathscr{F}} \int_{0}^{t}\left\|e^{-A \lambda_{n}^{2}(t-s)}\right\|\left\|T_{n}(s) X_{n}(x)\right\| d s\right)^{2} \\
\quad \leq\left(\sum_{\lambda_{n} \in \mathscr{F}}\left(\int_{0}^{t}\left\|e^{-A \lambda_{n}^{2}(t-s)}\right\|^{2} d s\right)^{1 / 2}\right. \\
\left.\times\left(\int_{0}^{t}\left\|T_{n}(s)\right\|^{2}\left|X_{n}(x)\right|^{2} d s\right)^{1 / 2}\right)^{2} .
\end{aligned}
$$

We define $a_{n}=\int_{0}^{t}\left\|e^{-A \lambda_{n}^{2}(t-s)}\right\|^{2} d s$, using inequality (42); it follows that

$$
a_{n}=\int_{0}^{t}\left\|e^{-A \lambda_{n}^{2}(t-s)}\right\|^{2} d s \leq \frac{L}{2 \lambda_{n}^{2} \alpha(A)}
$$

and as series $\sum_{\lambda_{n} \in \mathscr{F}}\left(1 / \lambda_{n}^{2}\right)$ is convergent, then series $\sum_{\lambda_{n} \in \mathscr{F}} a_{n}$ is also convergent. We define $b_{n}=\int_{0}^{t}\left\|T_{n}(s)\right\|^{2}\left|X_{n}(x)\right|^{2} d s$; it follows that

$$
\begin{aligned}
& \left\|T_{n}(s)\right\|^{2}\left|X_{n}(x)\right|^{2}=\left\|T_{n}(s)\right\|^{2}\left\|X_{n}(x)\right\|^{2} \frac{\left|X_{n}(x)\right|^{2}}{\left\|X_{n}(x)\right\|^{2}} ; \\
& \frac{\left|X_{n}(x)\right|^{2}}{\left\|X_{n}(x)\right\|^{2}} \\
& \quad=\frac{\left|X_{n}(x)\right|^{2}}{\int_{0}^{1} X_{n}^{2}(x) d x} \\
& \leq\left(\lambda_{n}^{2} b_{1}^{2}+\left|1-\rho_{0} b_{1}\right|^{2}+2\left|b_{1}\right|\left|1-\rho_{0} b_{1}\right| \lambda_{n}\right)
\end{aligned}
$$




$$
\begin{gathered}
\times\left(\frac{\lambda_{n}^{2} b_{1}^{2}}{2}-\frac{b_{1}\left(1-\rho_{0} b_{1}\right)}{2}+\frac{\left(1-\rho_{0} b_{1}\right)^{2}}{2}\right. \\
+\frac{\lambda_{n}^{2} b_{1}^{2}-\left(1-\rho_{0} b_{1}\right)^{2}}{4 \lambda_{n}} \sin \left(2 \lambda_{n}\right) \\
\left.+\frac{b_{1}\left(1-\rho_{0} b_{1}\right)}{2} \cos \left(2 \lambda_{n}\right)\right)^{-1} \\
=A\left(\lambda_{n}\right),
\end{gathered}
$$

using (26) one gets that

$$
\lim _{n \rightarrow \infty} A\left(\lambda_{n}\right)=2
$$

then there exists a positive integer $n_{0} \in \mathbb{N}$ so that, for all index $n$ so that $\lambda_{n} \in \mathscr{F}$ and $n>n_{0}$, one gets that

$$
\frac{\left|X_{n}(x)\right|^{2}}{\left\|X_{n}(x)\right\|^{2}}<3
$$

and replacing in (46),

$$
\begin{array}{r}
\left\|T_{n}(s)\right\|^{2} X_{n}^{2}(x) \leq 3\left\|T_{n}(s)\right\|^{2}\left\|X_{n}(x)\right\|^{2}, \\
n \in\left\{m \in \mathbb{N}: \lambda_{m} \in \mathscr{F}, m>n_{0}\right\} .
\end{array}
$$

Applying Bessel's inequality (25), it follows that

$$
\begin{aligned}
\sum_{\lambda_{n} \in \mathscr{F}}\left\|T_{n}(s)\right\|^{2}\left|X_{n}(x)\right|^{2} & \leq 3 \sum_{\lambda_{n} \in \mathscr{F}}\left\|T_{n}(s)\right\|^{2}\left\|X_{n}(x)\right\|^{2} \\
& \leq 3 \int_{0}^{1}\|G(x, s)\|^{2} d x .
\end{aligned}
$$

This ensures that the series $\sum_{\lambda_{n} \in \mathscr{F}}\left\|T_{n}(s)\right\|^{2}\left|X_{n}(x)\right|^{2}$ is uniformly convergent and integrating in the interval $[0, t], t \geq 0$; therefore,

$$
\sum_{\lambda_{n} \in \mathscr{F}} \int_{0}^{t}\left\|T_{n}(s)\right\|^{2}\left|X_{n}(x)\right|^{2} d s \leq 3 \int_{0}^{t} \int_{0}^{1}\|G(x, s)\|^{2} d x d s
$$

where, for a fixed value of $t \in[c, d]$, the positive terms series $b_{n}$ has the partial sum bounded if we suppose that vector valued function $G(x, t)$ satisfies the following condition:

$$
\sup _{t>0} \int_{0}^{1}\|G(x, t)\|^{2} d x=M<\infty .
$$

If condition (53) holds, series $\sum_{n / \lambda_{n} \in \mathscr{F}} b_{n}$ is convergent. Using (21), (42), and (52) in (44), it follows that

$$
\begin{aligned}
\left\|\sum_{\lambda_{n} \in \mathscr{F}} e^{-\lambda_{n}^{2} A t} X_{n}(x) B_{n}(t)\right\|^{2} \\
\leq\left(\sum_{\lambda_{n} \in \mathscr{F}} \int_{0}^{t}\left\|e^{-A \lambda_{n}^{2}(t-s)}\right\|^{2} d s\right) \\
\quad \times\left(\sum_{\lambda_{n} \in \mathscr{F}} \int_{0}^{t}\left\|T_{n}(s)\right\|^{2} X_{n}^{2}(x) d s\right) \\
\leq \frac{3 L}{2 \alpha(A)}\left(\int_{0}^{t} \int_{0}^{1}\|G(x, s)\|^{2} d x d s\right) \sum_{\lambda_{n} \in \mathscr{F}} \frac{1}{\lambda_{n}^{2}},
\end{aligned}
$$

and taking into account that $\sum_{\lambda_{n} \in \mathscr{F}}\left(1 / \lambda_{n}^{2}\right)$ is convergent, series $\sum_{\lambda_{n} \in \mathscr{F}} e^{-\lambda_{n}^{2} A t} X_{n}(x) B_{n}(t)$ is uniformly convergent on any domain $[0,1] \times[c, d]$.

To check that solution $w(x, t)$ given in (35) is a solution of problem (13)-(16), it is sufficient to show that the series

$$
\sum_{\lambda_{n} \in \mathscr{F}} \lambda_{n}^{2} e^{-\lambda_{n}^{2} A t} X_{n}(x) B_{n}(t)
$$

is uniformly convergent. To prove this, note that $G(x, t)$ satisfies the boundary condition (14) and (15); then,

$$
\int_{0}^{1} \frac{\partial^{2}}{\partial x^{2}}(G(x, t)) X_{n}(x) d x=-\lambda_{n}^{2} \int_{0}^{1} G(x, t) X_{n}(x) d x,
$$

And, by (24), one gets that

$$
\lambda_{n}^{2} T_{n}(t)\left\|X_{n}(x)\right\|^{2}=-\int_{0}^{1} G_{x x}(x, t) X_{n}(x) d x ;
$$

Then, if the following condition

$$
\sup _{t>0} \int_{0}^{1}\left\|G_{x x}(x, t)\right\|^{2} d x=N<\infty
$$

holds, the convergence of the series (55) can be derived in the same way as the convergence of the series $\sum_{\lambda_{n} \in \mathscr{F}} e^{-\lambda_{n}^{2} A t} X_{n}(x) B_{n}(t)$ has been deduced, and, thus, series $(55)$ is uniformly convergent on any domain $[0,1] \times[c, d]$. Summarizing, the following result has been established.

Theorem 1. Consider a be nonhomogeneous problem with homogeneous boundary values conditions (13)-(15) which satisfies conditions (7)-(9). Suppose that hypotheses of Theorem 2 of [15] hold, then we can construct a solution $v(x, t)$ of homogeneous problem with homogeneous boundary values conditions (34). Suppose that $G(x, t)$ satisfies conditions (8) replacing $f(x)$ by $G(x, t)$ and satisfies conditions (53) and (58). Then, $w(x, t)$, defined by (35), is a solution of nonhomogeneous problem with homogeneous boundary values conditions (36), and the solution of problem (13)-(15) is given by $u(x, t)=$ $v(x, t)+w(x, t)$. 


\section{A Series Solution for Nonhomogeneous Problem (13)-(16) under Hypotheses (10)-(12). Convergence}

We will suppose that the vector valued function $G(x, t)$ satisfies conditions (10) replacing $f(x)$ by $G(x, t)$, and, therefore, $G(x, t)$ admits a series expansion of Sturm-Liouville eigenfunctions which is given by

$$
G(x, t)=X_{0}(x) \widetilde{T}_{0}(t)+\sum_{\lambda_{n} \in \mathscr{F}} \sin \left(\lambda_{n} x\right) \widetilde{T}_{n}(t),
$$

where

$$
\begin{gathered}
\widetilde{T}_{n}(t)=\frac{\int_{0}^{1} G(x, t) \sin \left(\lambda_{n} x\right) d x}{\int_{0}^{1} \sin ^{2}\left(\lambda_{n} x\right) d x}, \\
\widetilde{T}_{0}(t)=\frac{\int_{0}^{1} G(x, t) x d x}{\int_{0}^{1} x^{2} d x} .
\end{gathered}
$$

Using again the eigenfunction method, we will construct a formal solution of the problem (13)-(15) in the form

$$
u(x, t)=X_{0}(x) \widetilde{R}_{0}(t)+\sum_{\lambda_{n} \in \mathscr{F}} e^{-A \lambda_{n}^{2} t} \sin \left(\lambda_{n} x\right) \widetilde{R}_{n}(t),
$$

where

$$
\begin{array}{cc}
\widetilde{B}_{n}(t)=\int_{0}^{t} e^{A \lambda_{n}^{2} s} \widetilde{T}_{n}(s) d s, & \widetilde{B}_{0}(t)=\int_{0}^{t} \widetilde{T}_{0}(s) d s, \\
\widetilde{R}_{n}(t)=\widetilde{B}_{n}(t)+\widetilde{D}_{n}, \quad \widetilde{R}_{0}(t)=\widetilde{B}_{0}(t)(t)+\widetilde{D}_{0},
\end{array}
$$

and as $f(x)$ satisfies (10), one gets

$$
\widetilde{D}_{n}=\frac{\int_{0}^{1} f(x) \sin \left(\lambda_{n} x\right) d x}{\int_{0}^{1} \sin ^{2}\left(\lambda_{n} x\right) d x}, \quad \widetilde{D}_{0}=\frac{\int_{0}^{1} f(x) x d x}{\int_{0}^{1} x^{2} d x} \text {. }
$$

Nothe that, as in Section 2, from (61) it follows that

$$
\begin{aligned}
u(x, t)= & X_{0}(x) \widetilde{R}_{0}(t) \\
& +\sum_{\lambda_{n} \in \mathscr{F}} e^{-\lambda_{n}^{2} A t} \sin \left(\lambda_{n} x\right) \widetilde{R}_{n}(t) \\
= & X_{0}(x) \widetilde{D}_{0}+\sum_{\lambda_{n} \in \mathscr{F}} e^{-\lambda_{n}^{2} A t} \sin \left(\lambda_{n} x\right) \widetilde{D}_{n} \\
& +X_{0}(x) \widetilde{B}_{0}(t)+\sum_{\lambda_{n} \in \mathscr{F}} e^{-\lambda_{n}^{2} A t} \sin \left(\lambda_{n} x\right) \widetilde{B}_{n}(t) \\
= & v(x, t)+w(x, t),
\end{aligned}
$$

where $v(x, t)$ is a solution of homogeneous problem with homogeneous boundary values conditions (34), whose convergence has been studied in [16]; we will study the convergence of $w(x, t)$ solution of problem (36), defined by

$$
w(x, t)=X_{0}(x) \widetilde{B}_{0}(t)+\sum_{\lambda_{n} \in \mathscr{F}} e^{-\lambda_{n}^{2} A t} \sin \left(\lambda_{n} x\right) \widetilde{B}_{n}(t),
$$

but this can be considered a special case of the one studied in Section 2 taking $b_{1}=0$. Thus, we have the following Theorem.
Theorem 2. Consider a be nonhomogeneous problem with homogeneous boundary values conditions (13)-(15) which satisfies conditions (10)-(12). Suppose that hypotheses of Theorem 3.1 of [16] hold; then, we can construct a solution $v(x, t)$ of homogeneous problem with homogeneous boundary values conditions (34). Suppose that vector valued function $G(x, t)$ satisfies conditions (10) replacing $f(x)$ by $G(x, t)$ and satisfies conditions (53) and (58). Then, $w(x, t)$, defined by (35), is a solution of nonhomogeneous problem with homogeneous boundary values conditions (36), and the solution of problem (13) $-(15)$ is given by $u(x, t)=v(x, t)+w(x, t)$.

\section{Algorithm and Examples}

We can summarize the process to calculate the solution of the problem (13)-(15) from Theorems 1 and 2 in Algorithm 1.

Example 3. We consider problem (13)-(15) where matrix $A \in$ $\mathbb{C}^{4 \times 4}$ is given by

$$
A=\left(\begin{array}{cccc}
2 & 0 & 0 & -1 \\
1 & 2 & 1 & -2 \\
-1 & 0 & 2 & 1 \\
0 & 0 & 0 & 1
\end{array}\right)
$$

and the matrices $A_{i}, B_{i}, i \in\{1,2\}$ are

$$
\begin{array}{ll}
A_{1}=\left(\begin{array}{llll}
0 & 0 & 0 & 0 \\
0 & 0 & 0 & 0 \\
0 & 0 & 1 & 0 \\
0 & 0 & 0 & 1
\end{array}\right), & A_{2}=\left(\begin{array}{llll}
0 & 1 & 0 & 0 \\
1 & 0 & 0 & 0 \\
0 & 0 & 0 & 1 \\
0 & 0 & 0 & 0
\end{array}\right) \\
B_{1}=\left(\begin{array}{llll}
1 & 0 & 0 & 0 \\
0 & 1 & 0 & 0 \\
0 & 0 & 0 & 0 \\
0 & 0 & 0 & 0
\end{array}\right), & B_{2}=\left(\begin{array}{llll}
1 & 0 & 0 & 0 \\
1 & 0 & 0 & 0 \\
0 & 0 & 1 & 0 \\
0 & 0 & 0 & 1
\end{array}\right) ;
\end{array}
$$

the vectorial valued function $f(x)$ will be defined as

$$
f(x)=\left(\begin{array}{c}
0 \\
x^{2}-1 \\
0 \\
0
\end{array}\right)
$$

and the vectorial valued function $G(x, t)$ is given by

$$
G(x, t)=\left(\begin{array}{c}
0 \\
(x-1)^{2} x^{3} e^{-t} \\
0 \\
0
\end{array}\right)
$$

We will follow Algorithm 1 step by step.

(1) If we consider the associated problem (34), it is easy to check that conditions (7)-(9) hold. In fact, this problem was solved in Example 3.1 of [15]. Using Algorithm 1 of [15], we can obtain the solution $v(x, t)$ 


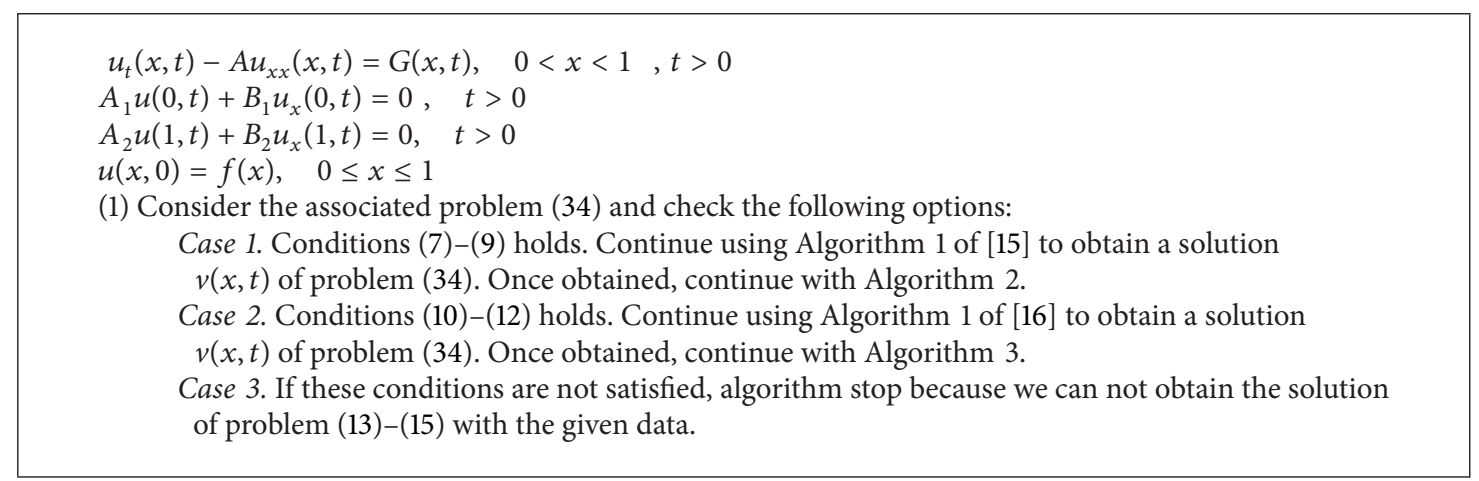

Algorithm 1: Solution of problem (13)-(15).

of problem (34) with the values $\rho_{0}=1, b_{1}=1, b_{2}=0$. The solution of problem (34) is given by

$$
\begin{aligned}
& v(x, t) \\
& =\left(\sum_{n \geq 0}-\frac{32(-1)^{n} e^{-(1 / 2)(\pi+2 n \pi)^{2} t} \cos ((1 / 2)(\pi+2 n \pi) x)}{\pi^{3}(2 n+1)^{3}}\right) \\
& \quad \times\left(\begin{array}{l}
0 \\
1 \\
0 \\
0
\end{array}\right),
\end{aligned}
$$

with the eigenvalues set $\mathscr{F}=\left\{\lambda_{k}=\pi / 2+k \pi: k \geq 0\right\}$ and eigenfunctions

$$
X_{k}(x)=-\left(\frac{\pi}{2}+k \pi\right) \cos \left(\left(\frac{\pi}{2}+k \pi\right) x\right), \quad k \geq 0 .
$$

After obtaining the solution of the homogeneous problem with homogeneous conditions (34), we continue with Algorithm 2.

We will follow Algorithm 2 step by step.

(1) It is trivial to check that, for fixed $t$,

$$
G(x, t) \in \mathscr{C}^{2}([0,1])
$$

Therefore,

$$
\begin{gathered}
\left(1-\rho_{0} b_{1}\right) G(0, t)+b_{1} G_{x}(0, t)=\left(\begin{array}{l}
0 \\
0 \\
0 \\
0
\end{array}\right), \\
-\left(\frac{1-b_{2}+\rho_{0} b_{1} b_{2}}{b_{1}}\right) G(1, t)+b_{2} G_{x}(1, t)=\left(\begin{array}{l}
0 \\
0 \\
0 \\
0
\end{array}\right) ;
\end{gathered}
$$

then, the vector valued function $G(x, t)$ satisfies condition (8) replacing $f(x)$ by $G(x, t)$. By other hand, one gets that

$$
\begin{aligned}
\int_{0}^{1}\|G(x, t)\|^{2} d x & =\int_{0}^{1}\left(e^{-2 t}(-1+x)^{4} x^{6}\right) d x \\
& =\frac{e^{-2 t}}{2310},
\end{aligned}
$$

and, thereby, $\sup _{t>0} \int_{0}^{1}\|G(x, t)\|^{2} d x \leq 1 / 2310=M$ and condition (53) holds. Similarly,

$$
\begin{aligned}
\int_{0}^{1} & \left\|G_{x x}(x, t)\right\|^{2} d x \\
& =\int_{0}^{1}\left(\left(6 e^{-t}(-1+x)^{2} x+12 e^{-t}(-1+x) x^{2}+2 e^{-t} x^{3}\right)^{2}\right) d x \\
& =\frac{12 e^{-2 t}}{35}
\end{aligned}
$$

and, thereby, $\sup _{t>0} \int_{0}^{1}\left\|G_{x x}(x, t)\right\|^{2} d x \leq 12 / 35=N$ and condition (58) holds.

(2) For $n \geq 0$, coefficients $T_{n}(t)$ defined by (24) are given by

$$
T_{n}(t)
$$

$$
\begin{aligned}
& =\left(\left(6 4 e ^ { - t } \left((-1)^{n}(2 n+1) \pi\left((2 n+1)^{2} \pi^{2}-144\right)\right.\right.\right. \\
& \left.\left.-6\left((2 n+1)^{2} \pi^{2}-80\right)\right)\right) \\
& \left.\times\left((2 n+1)^{7} \pi^{7}\right)^{-1}\right)
\end{aligned}
$$


(1) Check that vector valued function $G(x, t)$ satisfies conditions (53), (58) and (8) replacing $f(x)$ by $G(x, t)$. If these conditions are not satisfied algorithm stop because we can not obtain the solution of problem (13)-(15) with the given data.

(2) Determine coefficients $T_{n}(t), n \geq 0$ defined by (24).

(3) Determine coefficients $B_{n}(t), n \geq 0$ defined by (28), where $D_{n}, n \geq 0$ are defined by (31).

(4) Determine $w(x, t)$ defined by (35), solution of problem (36).

(5) The solution of problem (13)-(15) is given by $u(x, t)=v(x, t)+w(x, t)$.

Algorithm 2: Algorithm to compute the solution of problem (13)-(15) when conditions (7)-(9) holds.

(3) For $n \geq 0$, coefficients $B_{n}(t)$ defined by (28) are given by

$$
\begin{aligned}
& B_{n}(t) \\
& =\left(\left(128\left(e^{\left(-2+(2 n+1)^{2} \pi^{2}\right) t / 2}-1\right)\right.\right. \\
& \quad \times\left((-1)^{n}(2 n+1) \pi\left((2 n+1)^{2} \pi^{2}-144\right)\right. \\
& \left.\left.\quad-6\left((2 n+1)^{2} \pi^{2}-80\right)\right)\right) \\
& \left.\times\left((2 n+1)^{7} \pi^{7}\left(-2+(2 n+1)^{2} \pi^{2}\right)\right)^{-1}\right) \\
& \times\left(\begin{array}{l}
0 \\
1 \\
0 \\
0
\end{array}\right) .
\end{aligned}
$$

(4) The solution $w(x, t)$ of problem (36) defined by (35) is given by

$$
\begin{aligned}
& w(x, t) \\
& =\sum_{n \geq 0}-\left(\left(64 e^{-(2 n+1)^{2} \pi^{2} t / 2}\left(e^{\left(-2+(2 n+1)^{2} \pi^{2}\right) t / 2}-1\right)\right.\right. \\
& \left.\quad \times \mathscr{A}(n) \cos \left(\frac{(2 n+1) \pi x}{2}\right)\right) \\
& \left.\times\left((2 n+1)^{6} \pi^{6}\left(-2+(2 n+1)^{2} \pi^{2}\right)\right)^{-1}\right) \\
& \times\left(\begin{array}{l}
0 \\
1 \\
0 \\
0
\end{array}\right)
\end{aligned}
$$

where

$$
\begin{aligned}
& \mathscr{A}(n) \\
& \begin{aligned}
=(480+(2 n+1) \pi( & -144(-1)^{n} \\
& \left.\left.+(2 n+1) \pi\left((-1)^{n}(2 n+1) \pi-6\right)\right)\right) .
\end{aligned}
\end{aligned}
$$

(5) The solution of problem (13)-(15) is given by $u(x, t)=$ $v(x, t)+w(x, t)$.
Example 4. We consider problem (13)-(15) where matrix $A \in$ $\mathbb{C}^{4 \times 4}$ is given by

$$
A=\left(\begin{array}{cccc}
2 & 0 & 0 & 1 \\
1 & 2 & 0 & -2 \\
-1 & 0 & 2 & 1 \\
0 & 0 & 0 & 1
\end{array}\right)
$$

and the matrices $A_{i}, B_{i}, i \in\{1,2\}$ are given by (68). The vectorial valued function $f(x)$ is defined by

$$
f(x)=\left(\begin{array}{c}
0 \\
0 \\
x^{2}-2 x \\
0
\end{array}\right)
$$

and the vectorial valued function $G(x, t)$ is given by

$$
G(x, t)=\left(\begin{array}{c}
0 \\
0 \\
x(x-1)^{2} e^{-t} \\
0
\end{array}\right)
$$

We will follow Algorithm 1 step by step.

(1) If we consider the associated problem (34), it is easy to check that conditions (10)-(12) hold. In fact, this problem was solved in Example 4.1 of [16]. Using Algorithm 1 of [16] we can obtain the solution $v(x, t)$ of problem (34) with the values $\rho_{0}=1, a_{2}=0$. The solution of problem (34) is given by

$v(x, t)$

$=\sum_{n \geq 0}-\frac{32 e^{-(1 / 2)(\pi+2 n \pi)^{2} t} \sin ((1 / 2)(1+2 k) \pi x)}{\pi^{3}(2 k+1)^{3}}$

$$
\times\left(\begin{array}{l}
0 \\
0 \\
1 \\
0
\end{array}\right)
$$

with the eigenvalues set $\mathscr{F}=\left\{\lambda_{k}=\pi / 2+k \pi: k \geq 0\right\}$ and eigenfunctions

$$
X_{k}(x)=\sin \left(\left(\frac{\pi}{2}+k \pi\right) x\right), \quad k \geq 0
$$


(1) Check that vector valued function $G(x, t)$ satisfies conditions (53), (58) and (10) replacing $f(x)$ by $G(x, t)$. If these conditions are not satisfied algorithm stop because we can not obtain the solution of problem (13)-(15) with the given data.

(2) Determine coefficients $\widetilde{T}_{n}(t), n \geq 0$ defined by (60).

(3) Determine coefficients $\widetilde{B}_{n}(t), n \geq 0$ defined by (62), where $\widetilde{D}_{n}, n \geq 0$ are defined by (64).

(4) Determine $w(x, t)$ defined by (66), solution of problem (36).

(5) The solution of problem (13)-(15) is given by $u(x, t)=v(x, t)+w(x, t)$.

Algorithm 3: Algorithm to compute the solution of problem (13)-(15) when conditions (10)-(12) holds.

After obtaining the solution of the homogeneous problem with homogeneous conditions (34), we continue with Algorithm 3.

We will follow Algorithm 3 step by step.

(1) We will check that the vector valued function $G(x, t)$ satisfies conditions (10) replacing $f(x)$ by $G(x, t)$.

It is trivial to check that, for fixed $t$,

$$
G(x, t) \in \mathscr{C}^{2}([0,1]) .
$$

Therefore,

$$
\begin{gathered}
G(0, t)=\left(\begin{array}{l}
0 \\
0 \\
0 \\
0
\end{array}\right), \\
a_{2} G(1, t)+G_{x}(1, t)=\left(\begin{array}{l}
0 \\
0 \\
0 \\
0
\end{array}\right) .
\end{gathered}
$$

Then, conditions (10) hold. Furthermore, one gets that

$$
\begin{aligned}
\int_{0}^{1}\|G(x, t)\|^{2} d x & =\int_{0}^{1}\left(e^{-2 t} x^{2}\left(x^{2}-1\right)^{4}\right) d x \\
& =\frac{128 e^{-2 t}}{3465} ;
\end{aligned}
$$

thus, $\sup _{t>0} \int_{0}^{1}\|G(x, t)\|^{2} d x \leq 128 / 3465=M<\infty$ and condition (53) holds.

Similarly,

$$
\begin{aligned}
& \int_{0}^{1}\left\|G_{x x}(x, t)\right\|^{2} d x \\
& =\int_{0}^{1}\left(\left(8 e^{-t} x\left(x^{2}-1\right)+e^{-t} x\left(8 x^{2}+4\left(x^{2}-1\right)\right)\right)^{2}\right) d x \\
& =\frac{64 e^{-2 t}}{7}
\end{aligned}
$$

thus, $\sup _{t>0} \int_{0}^{1}\left\|G_{x x}(x, t)\right\|^{2} d x \leq 64 / 7=N<\infty$ and condition (58) holds.
(2) For $n \geq 0$, coefficients $\widetilde{T}_{n}(t)$ defined by (60) are given by

$$
\widetilde{T}_{n}(t)=-\frac{1536(-1)^{n} e^{-t}\left(-10+(2 n+1)^{2} \pi^{2}\right)}{(2 n+1)^{6} \pi^{6}}\left(\begin{array}{l}
0 \\
0 \\
1 \\
0
\end{array}\right)
$$

(3) For $n \geq 0$, coefficients $\widetilde{B}_{n}(t)$ defined by (62) are given by

$$
\begin{aligned}
& \widetilde{B}_{n}(t) \\
& =-\frac{3072(-1)^{n}\left(e^{(1 / 2)\left(-2+(2 n+1)^{2} \pi^{2}\right) t}-1\right)\left((2 n+1)^{2} \pi^{2}-10\right)}{(2 n+1)^{6} \pi^{6}\left(-2+(2 n+1)^{2} \pi^{2}\right)} \\
& \quad \times\left(\begin{array}{l}
0 \\
0 \\
1 \\
0
\end{array}\right) .
\end{aligned}
$$

(4) The solution $w(x, t)$ of problem (36), defined by (66), is given by

$$
\begin{aligned}
& w(x, t) \\
& =\sum_{n \geq 0}-\left(\left(3072(-1)^{n} e^{-(2 n+1)^{2} \pi^{2} t / 2}\left(e^{\left(-2+(2 n+1)^{2} \pi^{2}\right) t / 2}-1\right)\right.\right. \\
& \left.\times\left((2 n+1)^{2} \pi^{2}-10\right) \sin \left(\frac{(2 n+1) \pi x}{2}\right)\right) \\
& \left.\times\left((2 n+1)^{6} \pi^{6}\left(-2+(2 n+1)^{2} \pi^{2}\right)\right)^{-1}\right) \\
& \times\left(\begin{array}{l}
0 \\
0 \\
1 \\
0
\end{array}\right) .
\end{aligned}
$$

(5) The solution of problem (13)-(15) is given by $u(x, t)=$ $v(x, t)+w(x, t)$. 


\section{Conclusions}

In this paper, the construction of the exact series solution of the nonhomogeneous problem (13)-(16) has been presented. Conditions for the vector valued function $G(x, t)$ in order to ensure the existence and convergence of a series solution of the proposed problem have been presented. An algorithm with two illustrative examples was given.

\section{Conflict of Interests}

The authors declare that there is no conflict of interests regarding the publication of this paper.

\section{References}

[1] M. H. Alexander and D. E. Manolopoulos, "A stable linear reference potential algorithm for solution of the quantum closecoupled equations in molecular scattering theory," The Journal of Chemical Physics, vol. 86, no. 4, pp. 2044-2050, 1986.

[2] V. S. Melezhik, I. V. Puzynin, T. P. Puzynina, and L. N. Somov, "Numerical solution of a system of integro-differential equations arising from the quantum mechanical three-body problem with Coulomb interaction," Journal of Computational Physics, vol. 54, no. 2, pp. 221-236, 1984.

[3] W. T. Reid, Ordinary Differential Equations, John Wiley \& Sons, New York, NY, USA, 1971.

[4] R. D. Levine, M. Shapiro, and B. R. Johnson, "Transition probabilities in molecular collisions: computational studies of rotational excitation," The Journal of Chemical Physics, vol. 52, no. 4, pp. 1755-1766, 1970.

[5] T. G. Schmalz, J. V. Lill, and J. C. Light, "Imbedded matrix Green's functions in atomic and molecular scattering theory," The Journal of Chemical Physics, vol. 78, no. 7, pp. 4456-4463, 1983.

[6] F. Mrugaía and D. Secrest, "The generalized log-derivative method for inelastic and reactive collisions," Journal of Chemical Physics, vol. 78, no. 10, pp. 5954-5961, 1983.

[7] J. Crank, The Mathematics of Diffusion, Oxford University Press, 2nd edition, 1995.

[8] M. D. Mikhailov and M. N. Osizik, Unifield Analysis and Solutions of Heat and Mass Diffusion, John Wiley \& Sons, New York, NY, USA, 1984.

[9] I. Stakgold, Green's Functions and Boundary Value Problems, John Wiley \& Sons, New York, NY, USA, 1979.

[10] T. Hueckel, M. Borsetto, and A. Peano, Modelling of Coupled Thermo-Elastoplastic Hydraulic Response of Clays Subjected to Nuclear Waste Heat, Wiley, New York, NY, USA, 1987.

[11] F. V. Atkinson, Discrete and Continuous Boundary Problems, Academic Press, New York, NY, USA, 1964.

[12] F. V. Atkinson, A. M. Krall, G. K. Leaf, and A. Zettel, "On the numerical computation of eigenvalues of Sturm-Liouville problems with matrix coefficients," Tech. Rep., Argonne National Laboratory, 1987.

[13] M. Marletta, Theory and implementation of algorithms for Sturm-Liouville systems [Ph.D. thesis], Royal Military College of Science, Cranfield, UK, 1991.

[14] L. Greenberg, "A Prufer method for calculating eigenvalues of self-adjoint systems of ordinary differential equations, parts 1 and 2," Tech. Rep. TR91-24, University of Maryland, 1991.
[15] V. Soler, E. Defez, and M. V. Ferrer, "On exact series solution of strongly coupled mixed parabolic problems," Abstract and Applied Analysis, vol. 2013, Article ID 524514, 9 pages, 2013.

[16] V. Soler, E. Defez, and J. A. Verdoy, "On exact series solution for strongly coupled mixed parabolic boundary value problems," Abstract and Applied Analysis, vol. 2014, Art. ID 759427, 9 pages, 2014.

[17] S. L. Campbell and C. D. Meyer Jr., Generalized Inverses, of Linear Transformations, Pitman, London, UK, 1979.

[18] E. Navarro, L. Jódar, and M. V. Ferrer, "Constructing eigenfunctions of strongly coupled parabolic boundary value systems," Applied Mathematics Letters, vol. 15, no. 4, pp. 429-434, 2002.

[19] G. H. Golub and C. F. van Loan, Matrix Computation, The Johns Hopkins University Press, Baltimore, Md, USA, 1989.

[20] I. S. Gradshteyn and I. M. Ryzhik, Tables of Integrals, Series and Products, Academic Press, 5th edition, 1980.

[21] G. H. Hardy, J. E. Littlewood, and G. Polya, Inequalities, Cambridge University Press, 1934.

[22] E. A. Coddington and N. Levinson, Theory of Ordinary Differential Equations, McGraw-Hill, New York, NY, USA, 1967. 


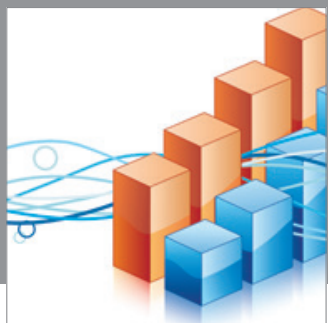

Advances in

Operations Research

mansans

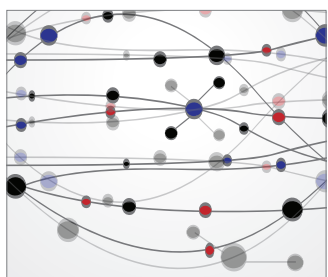

The Scientific World Journal
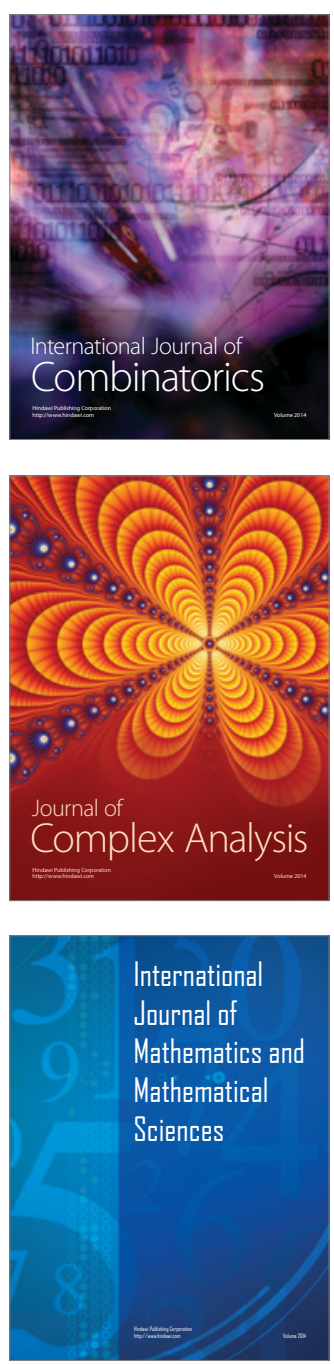
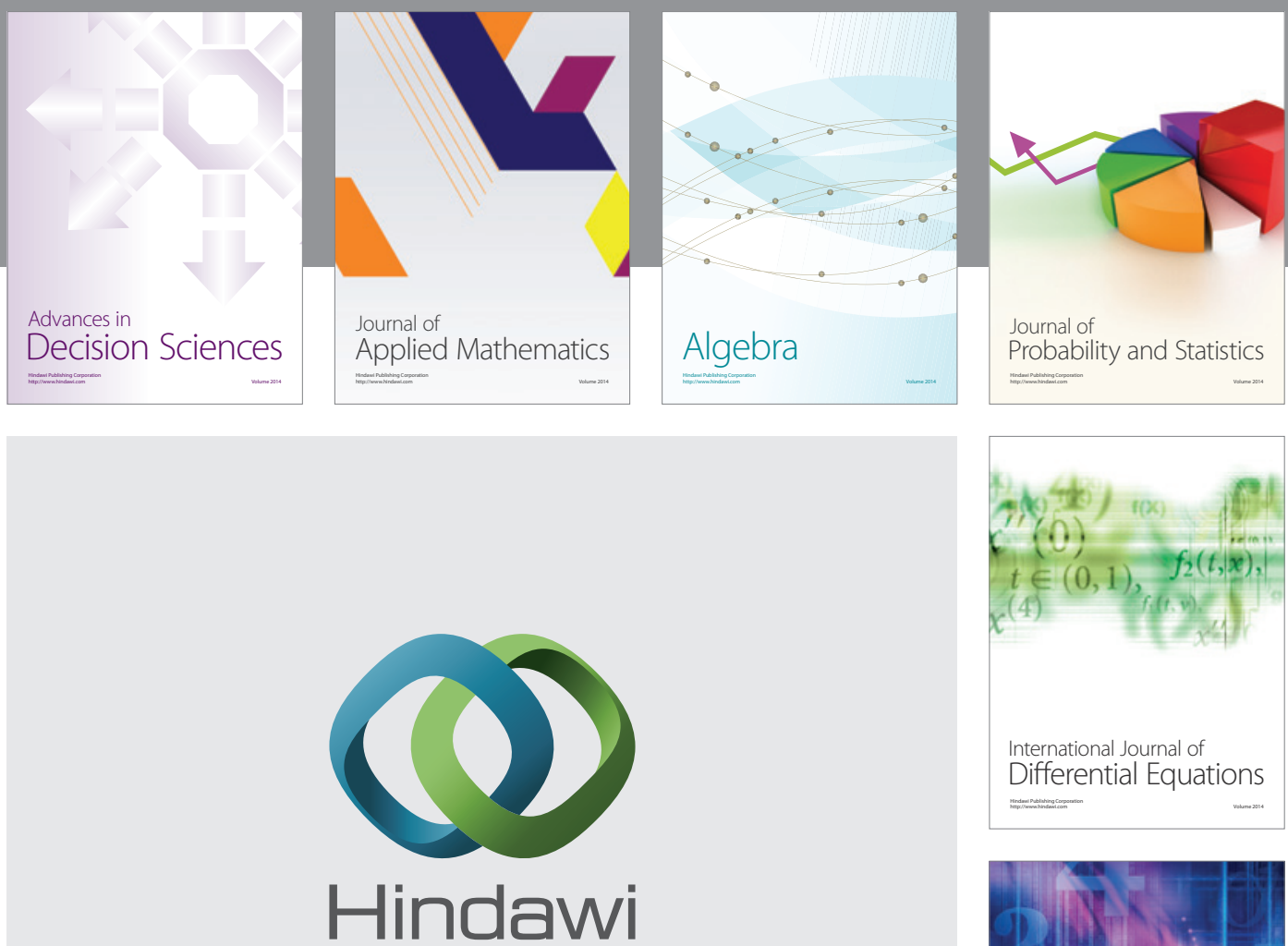

Submit your manuscripts at http://www.hindawi.com
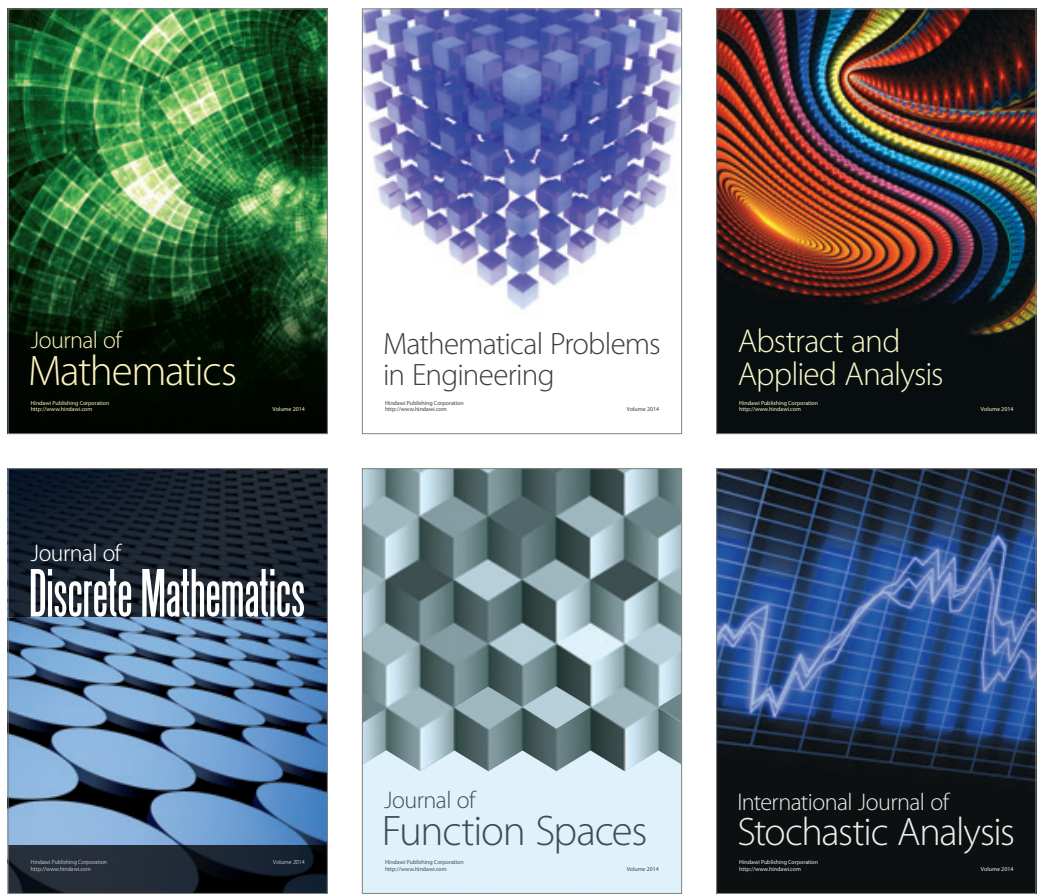

Journal of

Function Spaces

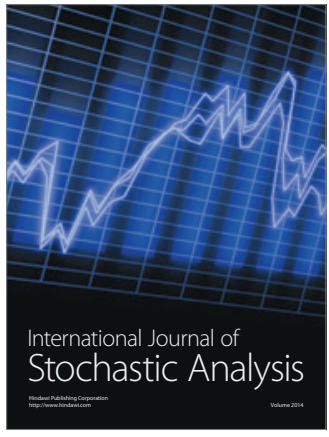

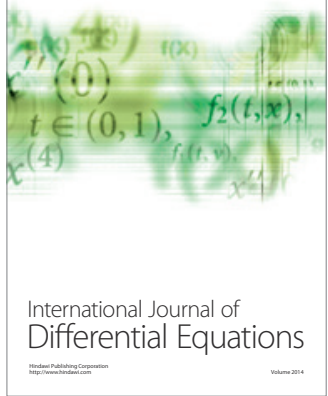
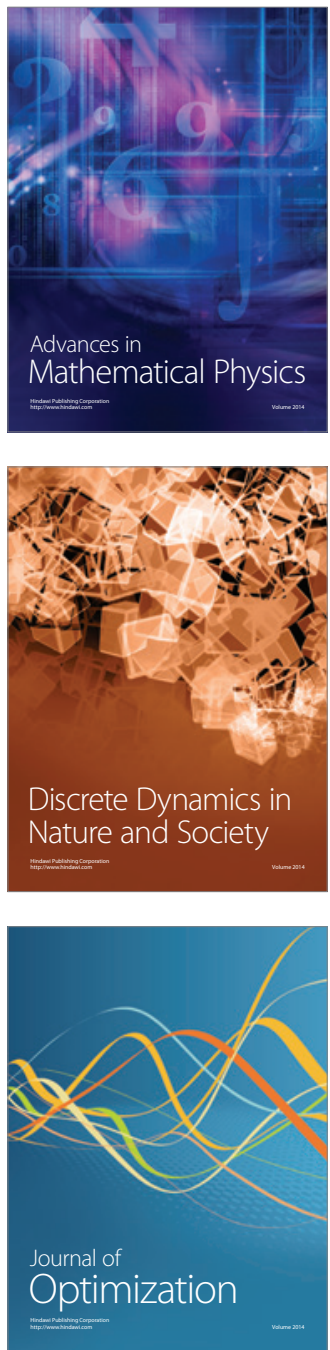